\title{
Propuesta de Innovación docente para el aprendizaje Interdisciplinar sobre Diseño para todas las personas entre estudiantes de los Grados de Odontología y de Terapia Ocupacional de diferentes Universidades españolas
}

\author{
Mónica Cano Rosása, María Yolanda González Alonso ${ }^{\mathrm{b}}$, Raquel Aceves Díez ${ }^{\mathfrak{c}}$, Thais \\ Pousada García ${ }^{\text {, }}$ Estíbaliz Jimenez Arberas ${ }^{\mathrm{e}}$ Emiliano Díez Villoría ${ }^{\text {af }}$ \\ aUniversidad de Salamanca, mcanorosas@usal.es, emid@usal.es buniversidad de Burgos, \\ mygonzalez@ubu.es, ${ }^{\mathrm{c}}$ Federación ASPACE CyL, federaspacecyl@gmail.com, ${ }^{\mathrm{d}}$ Universidad de La \\ Coruña, tpousada@udc.es, 'Universidad de Oviedo, estibaliz@,facultadpadreosso.es, fInstituto \\ Universitario de Integración en la Comunidad - INICO
}

\begin{abstract}
Teaching "design for all people" to students of Health Sciences is vital so that in the future they will be able to "design products and environments that are easy to use for as many people as possible, without the need to adapt or redesign them. a special way. " For this reason, during the first years of implementation of the degree titles, different types of actions have been proposed to include curricular contents on design for all people in very varied ways. For example, through specific subjects or crosscurricular approaches that spread the teaching in different subjects. The general objective of the project has been to design a set of activities of interdisciplinary nature among students of the Degree of Dentistry and the Degree of Occupational Therapy of several Spanish universities to exchange information and experiences in relation to the application of design principles for all people.
\end{abstract}

Keywords: Interdisciplinary learning, occupational therapy, dentistry, design for all

\section{Resumen}

Enseñar "diseño para todas las personas" a los estudiantes de Ciencias de la Salud es vital para que en el futuro sean capaces de "diseñar productos y entornos de fácil uso para el mayor número de personas posible, sin la necesidad de adaptarlos o rediseñarlos de una forma especial". Por eso, durante los primeros años de implantación de los títulos de grado, se han venido planteando distintos tipos de acciones dirigidas a incluir contenidos curriculares sobre diseño para todas las personas de maneras muy variadas. Por ejemplo, mediante asignaturas específicas o con planteamientos curriculares transversales que reparten la enseñanza en distintas asignaturas. El objetivo general del proyecto ha sido diseñar un conjunto de actividades de carácter interdisciplinar entre estudiantes del Grado de Odontología y del Grado de Terapia Ocupacional de varias universidades españolas para intercambiar información y experiencias en relación a la aplicación de los principios del diseño para todas las personas. 
Propuesta de Innovación docente para el aprendizaje Interdisciplinar sobre Diseño para todas las personas entre estudiantes de los Grados de Odontología y de Terapia Ocupacional de diferentes Universidades españolas

Palabras Clave: Aprendizaje interdisciplinar, terapia ocupacional, odontología, diseño para todas las personas.

\section{Introducción}

En general, se puede plantear que la enseñanza del Diseño para Todas las Personas ( DTP) debe estar guiada por una mezcla de prácticas pedagógicas tradicionales y alternativas, en las que el papel del profesor no es tanto aportar conocimientos expertos como ser capaz de ayudar a los estudiantes a identificar y utilizar fuentes de información apropiadas para aplicar el DTP a la práctica de su propia disciplina (Morrow, 2002).

Existe la necesidad de recibir formación específica relacionada con la inclusión (Collins, 2014; Díez, 2013; Sánchez y Díez, 2013) y diferenciar entre diseño universal y accesibilidad (Sánchez y Díez, 2016; Steinfeld y Maisel, 2012).

\section{Objetivo}

Se planteó el objetivo general de diseñar un conjunto de actividades de aprendizaje, de carácter interdisciplinar que permitiera a estudiantes de Terapia Ocupacional y de Odontología intercambiar información y experiencias en relación a la aplicación de los principios del diseño para todas las personas.

Se planteó el desarrollo de actividades de aprendizaje colaborativo que sirviesen para la adquisición de competencias de diseño para todas las personas y en las que se tratasen cuestiones como la comunicación con los pacientes y el uso de sistemas aumentativos y alternativos, el análisis accesibilidad física y del entorno en clínicas de odontología, la realización de adaptaciones y productos de apoyo para odontología o la educación y enseñanza de hábitos en pacientes odontológicos con discapacidad.

\section{Desarrollo de la innovación}

Las actividades se llevaron a cabo durante el segundo semestre del curso 2016-2017 en aulas de la Facultad de Psicología y en la Clínica Odontológica de la Facultad de Medicina de la Universidad de Salamanca, así como en aulas de Terapia Ocupacional en la Universidad de A Coruña.

El diseño para todas la personas es de gran relevancia para el desarrollo profesional del terapeuta ocupacional (Dejager y Lonnay, 2015; Lid, 2014; Liu, 2014; Ringaert, 2002) y para el odontólogo (Marulanda, Betancur, Espinosa, Gómez y Tapias, 2011). Y deben contemplarse actividades de aprendizaje interdisciplinar en las que interactúen diferentes profesionales implicados en la implementación del diseño para todas las personas (Larkin, Hitch, Watchorn, Ang y Stagnitti, 2013; Preiser y Smith, 2011; Watchorn, Larkin, Ang y Hitch, 2013).

A continuación, se resumen las principales actividades realizadas: 


\subsection{Actividad 1: Definición de competencias y resultados de aprendizaje}

Para definir los aspectos esenciales y el modo de integrarlo en las competencias y resultados de aprendizaje de las titulaciones implicadas en el proyecto (Terapia Ocupacional y Odontología) se consultaron las guías de Formación Curricular en Diseño para todas las personas de las titulaciones de Terapia Ocupacional (Díez et al., 2016) y Medicina (Mirón, Coco, Alonso, Maldonao e Iglesias, 2014), esta última por no estar disponible aún la guía de Odontología. Ambos documentos ofrecen información específica y de interés para determinar los objetivos de las actividades que se pretendían diseñar con este proyecto. Así que a partir de esa revisión se seleccionaron un conjunto de objetivos generales que debían satisfacer las actividades a diseñar y que pudieran ser aplicables a las dos titulaciones.

En el caso de Terapia Ocupacional:

- Adquirir nociones básicas sobre la diversidad del funcionamiento humano y cómo se ve afectado por distintas condiciones de salud, discapacidad o edad.

- Tener un conocimiento avanzado, y basado en la evidencia, sobre los beneficios sociales y terapéuticos derivados de la aplicación del Diseño para Todas las Personas.

- Ser capaz de evaluar productos y diseños atendiendo a la usabilidad para todas las personas y a su adherencia al paradigma de inclusión.

- Ser capaz de colaborar con administraciones nacionales y regionales, asociaciones profesionales y organizaciones relacionadas con la salud y los servicios sociales comunitarios, para promover la aplicación y evaluación de los principios del Diseño para Todas las Personas en entornos, productos y servicios.

- Ser capaz de concienciar y educar a consumidores y gestores mediante recursos sobre Diseño para Todas las Personas basados en la evidencia.

En el caso de Odontología (a partir de la guía de Medicina), estos son los objetivos elegidos:

- Dar a conocer a los universitarios los conceptos de diversidad, discapacidad y deficiencia, y la repercusión e impacto de estos términos en los ámbitos profesionales, culturales y sociales.

- Ser capaces de abordar la discapacidad a través de la identificación con la misma, de comunicarse de manera efectiva con personas con discapacidad, y de atender con los mismos niveles de calidad y seguridad que al resto de pacientes. Ser capaz de valorar la riqueza de lo diverso en cuanto a hábitos, culturas o costumbres, a fin de buscar soluciones integradoras o soluciones específicas, siempre desde el respeto a la diversidad.

Teniendo en cuenta estos objetivos se pasó a determinar la metodología docente a utilizar y el propio diseño de actividades.

\subsection{Actividad 2: Determinación de metodologías docentes y diseño de actividades}

En esta fase se revisaron trabajos como el de Welch (1995), Dyer, Callanan y Fennell (2010) o el editado por Christophersen (2002), Universal Design: 17 ways of thinking and teaching, 
Propuesta de Innovación docente para el aprendizaje Interdisciplinar sobre Diseño para todas las personas entre estudiantes de los Grados de Odontología y de Terapia Ocupacional de diferentes Universidades españolas

en el que se compilan distintas experiencias sobre inclusión de Diseño Universal en universidades de diferentes países de Europa, Norte América, Asia y Australia, que también podría servir de ayuda en el ámbito de la Terapia Ocupacional y la Odontología. Se muestran posibles áreas temáticas y estrategias docentes que se valoraron por parte del equipo de trabajo (Tabla 1).

Tabla 1. Áreas temáticas, técnicas de diseño y estrategias docentes en la enseñanza del diseño universal

\section{Áreas temáticas}

- Diversidad humana

- Reconocimiento de las múltiples formas de identidad

- Diseño de la Vida Cotidiana

- Relaciones personaentorno

- Capacidades Humanas (capacidades sensoriales, capacidades cognitivas, capacidades psicomotoras)

- Forma y Dimensiones Humanas

- Ergonomía

- Antropometría

- Diseño a lo largo del ciclo vital

\section{Técnicas de Diseño}

- Los 7 Principios del Diseño Universal

- Diseño mediante narraciones

- Diseño a lo largo del ciclo vital (diseño que tiene en cuenta las necesidades cambiantes de las personas en todos los estadios de su ciclo vital)

- Usuarios extremos

- Simulaciones

- Enfoque de la Pirámide del Diseño

- Análisis de patrones de diseño

- Listas de comprobación

- Evaluación a posteriori

- Participación del usuario (entrevistas, grupos focales, estudios observacionales, grabaciones en vídeo)

- Diseño para Rehabilitación

- Diseño para barreras o déficit específicos

\section{Estrategias Docentes}

- Clase magistral

- Taller (Presentaciones con debate)

- Proyecto (Trabajo dirigido por el estudiante)

- Estudio de Diseño (Proyectos dirigidos por el estudiante con espacios y equipos de apoyo)

- Implicación de agentes externos (miembros de la comunidad -personas mayores, niños, personas con discapacidad-; Profesionales - externos que asesoran y valoran críticamente los proyectos del estudiante-)

- Estrategias variadas (simulaciones, tutorías, uso de recursos multimedia)

Fuente: adaptado de Dyer, Callanan y Fennell, 2010)

Previamente al diseño de actividades se mantuvieron varias reuniones virtuales entre los profesionales implicados en el proyecto, para concretar los objetivos, las metodologías a aplicar y la forma de aplicación de estas.

En dichos encuentros se definieron cinco núcleos temáticos a desarrollar con la elaboración y puesta en marcha del presente proyecto:

- Introducción y aspectos básicos del Diseño para Todas las Personas.

- Análisis accesibilidad física y del entorno en clínicas de odontología.

- Productos de apoyo y adaptaciones en odontología.

- Educación y enseñanza de hábitos en pacientes odontológicos con diversidad funcional.

- Comunicación con los pacientes y el uso de sistemas aumentativos y alternativos.

Durante todo el proceso el equipo de trabajo tuvo en cuenta que las actividades deberían ser igualmente válidas para estudiantes de las dos titulaciones (Terapia Ocupacional y Odontología). Es decir, sin una profundización específica en contenidos propios de ambas 
disciplinas, sino con información y documentación más genérica, que permitan un trabajo coordinado y complementario entre alumnos/as.

Aunque no han llegado a diseñarse en su totalidad todas las actividades planificadas, y algunas aún están en desarrollo, el proyecto sí ha permitido completar el diseño y aplicación de algunas de las actividades. En resumen, estas son las actividades finalizadas y aplicadas, clasificadas en función de las áreas temáticas a que corresponden:

- Introducción y aspectos básicos del Diseño para Todas las Personas.

- Creación de una página Web en studium con tutoriales y documentos relacionados con el aprendizaje de competencias básicas sobre Diseño para Todas las personas. $\mathrm{Su}$ desarrollo se encuentra en una fase avanzada aunque aún en fase de diseño.

- Análisis accesibilidad física y del entorno en clínicas de odontología.

- Productos de apoyo y adaptaciones en odontología. Diseño y puesta en marcha de un taller compartido entre estudiantes de Terapia ocupacional y Odontología sobre productos de apoyo de bajo coste para Odontología.

- Educación y enseñanza de hábitos en pacientes odontológicos con diversidad funcional. Diseño y puesta en marcha de un taller compartido entre estudiantes de Terapia Ocupacional y Odontología sobre medición de placa bacteriana y técnicas de cepillado.

- Comunicación con los pacientes y el uso de sistemas aumentativos y alternativos. Diseño de un taller de aprendizaje colaborativo basado en un caso. Las estrategias estarían diseñadas para su aplicación, tanto en el aula, como en un medio virtual, con la posibilidad de obtener respuestas medibles de variables como participación, interacción, o nivel de adquisición de competencias.

\subsection{Actividad 3: Puesta en marcha de las actividades de aprendizaje con estudiantes de}

\section{Terapia Ocupacional y Odontología}

Algunas de las actividades diseñadas se pusieron en marcha durante el segundo semestre del curso 2016-2017 en la Universidad de Salamanca, en asignaturas de Terapia Ocupacional y Odontología, y en la Universidad de A Coruña, en una asignatura del Grado en Terapia Ocupacional. Durante el curso 2017-2018 comenzaon las actividades colaborativas entre asignaturas de Terapia Ocupacional en la Universidad de Burgos y Odontología en la Universidad de Salamanca. Cuando en próximos cursos las actividades de carácter interactivo estén finalizadas por completo se ha previsto que se realicen de manera coordinada entre los 
Propuesta de Innovación docente para el aprendizaje Interdisciplinar sobre Diseño para todas las personas entre estudiantes de los Grados de Odontología y de Terapia Ocupacional de diferentes Universidades españolas

estudiantes de esas asignaturas de las Universidades participantes desde el principio del cuatrimestre.

A continuación, se describirán las actividades diseñadas que se han puesto en marcha.

2.3.1. Creación de una página Web en studium con tutoriales y documentos relacionados con el aprendizaje de competencias básicas sobre Diseño para Todas las personas

Se ha comenzado el diseño de una página con recursos y actividades de carácter interactivo dirigidas a la adquisición de competencias básicas en diseño para todas las personas. El diseño de la página se encuentra en desarrollo y sigue los principios del diseño para todas las personas (medidas de accesibilidad Web, contenidos en formatos alternativos y accesibles, vídeos subtitulados, descritos y con transcripciones), (Figura 1).

$\equiv$ INICO ESPAÑOL - INTERNACIONAL (ES) -

\section{Diseño Universal en Terapia Ocupacional y Odontología}

Página Principal / Cursos / Formación interna INICO / DUTOOD

\section{General}

呾 Avisos

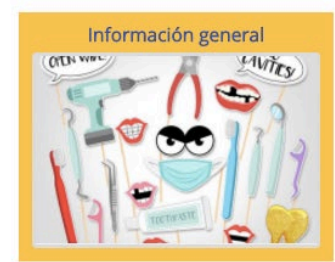

Productos de apoyo y adaptacio..

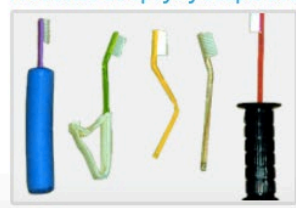

¿Qué es el Diseño Universal?

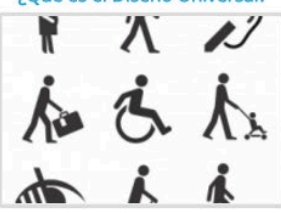

Comunicación aumentativa y al...

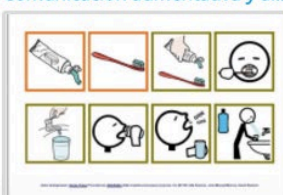

Accesibilidad

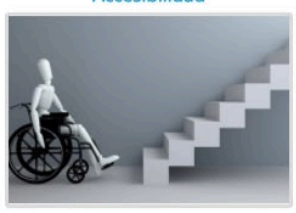

Higiene bucodental en paciente...

ร

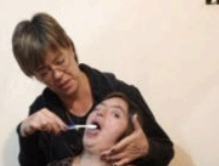

Figura 1. Página principal del sitio Moodle con actividades y recursos sobre Diseño para todas las personas (Diseño Universal) en Terapia Ocupacional y Odontología. En desarrollo

Se han realizado sesiones presenciales con estudiantes de las dos disciplinas en el marco de dos de los talleres diseñados a partir del desarrollo del proyecto.

(c)) BY-NC-ND 2019, Universitat Politècnica de València 
2.3.2. Taller sobre productos de apoyo de bajo coste en una clase de Terapia Ocupacional con participación de estudiantes de Odontología

En la literatura científica se encontran diversos estudios que concluyen que la higiene bucodental es muy deficiente y pobre en personas con discapacidad (Rao, Amitha, y Kishan, 2005). Una de las causas más notables para esta pobre higiene en la cavidad oral con sus consecuencias derivadas de la misma, radica en que un alto porcentaje de cuidadores y familiares no tienen conocimientos ni se les ha entrenado por parte de profesionales en esta tarea. Es necesario fomentar la prevención de enfermedades bucales en este colectivo mediante el entrenamiento tanto a la persona con discapacidad como a los cuidadores de la misma, y además enseñar en adaptaciones y posibles productos para llevar a cabo una correcta higiene bucodental (Barros, Mendes, Prado, y Machado, 2011; Medeiros, Brindeiro, Feitosa, y Wilney, 2011).

Son varios los estudios que indagan sobre el uso específico de los cepillos de dientes, aunque cabe señalar el estudio de Shaw, Shaw, y Foster (1989), los cuales evaluaron el manejo del cepillado y la habilidad de 75 niños en relación a la edad y género. Los tipos de agarre preferidos fueron el distal (73\%), fuerza (43\%) y oblicuo (29\%). Otro estudio fue el realizado por Beals, Wong, Allen, y Rutter (1999), que evaluaron a 45 niños y encontraron que el 43\% de niños cepillaron sus dientes con más de un tipo de agarre. Este hecho es importante puesto que dependiendo del tipo de agarre emplearemos una adaptación diferente para que la persona realice la actividad de manera autónoma.

\section{¿Por qué es importante el uso de adaptaciones para la higiene bucodental?}

Actualmente muchas personas con discapacidad encuentran limitaciones en la realización de diversas actividades de la vida diaria, entre las que destaca la higiene bucodental. Esta actividad ha sido muy poco valorada en este colectivo y una mala práctica de ella tiene consecuencias muy graves para la salud. Esta infravaloración es debida en parte al desconocimiento existente de productos de apoyo y adaptaciones de bajo coste. De aquí se deriva la importancia de conocer su utilidad, cómo confeccionarlas y cómo entrenar a la persona con discapacidad o al cuidador sobre su uso (Díez, Pousada y Perira, 2013).

El alumnado de segundo grado de Terapia Ocupacional y algunos estudiantes de segundo de Odontología, acudieron durante el curso académico 2017-2018 a un taller celebrado en la Universidad de Salamanca, en la Facultad de Psicología, a la asignatura de Adaptación funcional, productos y tecnologías de apoyo y ergonomía. Durante este taller los estudiantes pudieron observar en directo la fabricación de productos de bajo coste realizados con los 
Propuesta de Innovación docente para el aprendizaje Interdisciplinar sobre Diseño para todas las personas entre estudiantes de los Grados de Odontología y de Terapia Ocupacional de diferentes Universidades españolas

materiales más empleados para realizar adaptaciones de los útiles para la higiene bucodental. Además, los estudiantes elaboraron diferentes adaptaciones en función de diversos casos clínicos.

El taller tuvo una duración de cuatro horas, repartidas en dos sesiones de dos horas, con grupos de estudiantes de unos 25 participantes cada uno. En dicho taller los alumnos/as pudieron aprovechar el conocimiento práctico adquirido y el valor del equipo multidisciplinar en una actividad de la vida diaria como es la higiene bucodental (Figura 2).
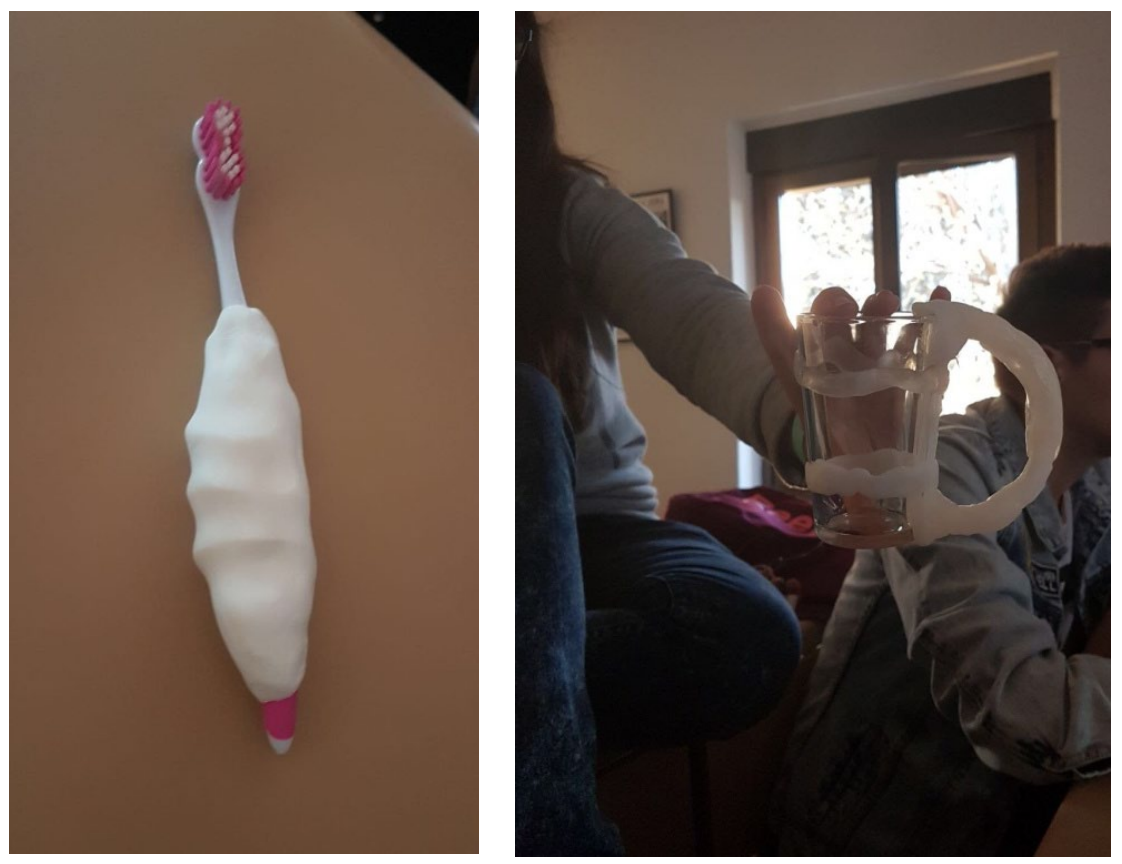

Figura 2. Detalle del taller sobre productos de apoyo de bajo coste. A la derecha un ejemplo de adaptación de cepillo de dientes con masilla de baja densidad. A la izquierda una adaptación para agarrar un vaso con material termoplástico.

2.3.3 Taller sobre medición de índice de placa bacteriana impartido por estudiantes de Odontología a estudiantes de Terapia Ocupacional

Se diseñó un proyecto de innovación docente con sesiones presenciales y sesiones on-line entre el alumnado de Odontología y el de Terapia Ocupacional con el objetivo de formarles en el campo preventivo de la salud oral. Las sesiones compartidas por todos los estuciantes se realizaron con el fin de establecer estrategias conjuntas para que, como futuros profesionales, puedan llevar a cabo un programa de control de placa bacteriana en pacientes crónicos y pacientes con discapacidad. 
La parte presencial del estudio se realizó en las instalaciones de la Clínica Odontológica de la facultad de Medicina de la Universida de Salanca, específicamente dispuestas para llevar a cabo un programa de control de placa bacteriana. El alumnado se agrupó de forma que con cada estudiantes de Odontología había dos estudiantes de Terapia Ocupacional.

Cada estudiante de Odontología instruyó a dos estudiantes de Terapia Ocupacional en técnicas de control de Placa Bacteriana mediante cepillado sobre fantomas.

\subsubsection{Diseño del Taller sobre comunicación alternativa}

Esta experiencia sólo se puso en marcha en la Universidad de A Coruña. En próximos cursos se pondrá en funcionamiento de manera conjunta entre estudiantes de las Universidades de Salamanca, de A Coruña, de Oviedo y de Burgos.

La unidad se planteó a partir de contenidos relacionados con la comunicación efectiva entre el profesional (odontólogo) y el paciente (usuario con diversidad funcional). Se destaca especialmente la información relacionada con la valoración, selección, entrenamiento y uso de los Sistemas Aumentativos y Alternativos de Comunicación (SAAC), y la aplicación de los principios del Diseño Universal en el proceso comunicativo. Se propone la realización de tres sesiones teórico - prácticas, complementadas con el trabajo no presencial por parte del alumnado.

\section{Resultados}

Al margen de los propios comentarios de satisfacción de los estudiantes que participaron en las actividades realizadas, a lo largo del curso y en las pruebas de evaluación de las asignaturas se introdujeron elementos para verificar el nivel de adquisición de contenidos sobre diseño para todas las personas.

En la evaluación inicial se utilizó un cuestionario tipo KPSI (Tabla 2). 
Propuesta de Innovación docente para el aprendizaje Interdisciplinar sobre Diseño para todas las personas entre estudiantes de los Grados de Odontología y de Terapia Ocupacional de diferentes Universidades españolas

Tabla 2. Resultados de actividad incial de autoevaluación sobre conocimientos respecto a contenidos relacionados con el diseño para todas las personas

\begin{tabular}{|c|c|c|c|c|c|c|}
\hline RESPUESTAS & $\begin{array}{l}\text { No lo } \\
\text { conozco/ } \\
\text { No lo } \\
\text { comprendo }\end{array}$ & $\begin{array}{l}\text { Lo conozco } \\
\text { un poco/ } \\
\text { Lo } \\
\text { comprendo } \\
\text { un poco }\end{array}$ & $\begin{array}{c}\text { Lo conozco } \\
\text { bastante } \\
\text { bien } / \text { Lo } \\
\text { comprendo } \\
\text { parcialmen } \\
\text { te }\end{array}$ & $\begin{array}{c}\text { Lo } \\
\text { comprendo } \\
\text { muy bien } \\
\text { aunque no } \\
\text { podría } \\
\text { explicarlo } \\
\text { a otra } \\
\text { personas }\end{array}$ & $\begin{array}{c}\text { Lo domino } \\
\text { de manera } \\
\text { que podría } \\
\text { explicárselo } \\
\text { a otra } \\
\text { persona }\end{array}$ & Total \\
\hline Ergonomía física & $17(77 \%)$ & $5(23 \%)$ & 0 & 0 & 0 & 22 \\
\hline Ergonomía cognitiva & $18(82 \%)$ & $4(18 \%)$ & 0 & 0 & 0 & 22 \\
\hline $\begin{array}{l}\text { Diseño Universal y } \\
\text { su implicación en } \\
\text { Terapia Ocupacional }\end{array}$ & $21(95 \%)$ & 0 & 0 & $1(5 \%)$ & 0 & 22 \\
\hline $\begin{array}{l}\text { Instumentos de } \\
\text { evaluación del } \\
\text { entorno }\end{array}$ & $10(45 \%)$ & $9(41 \%)$ & $2(9 \%)$ & $1(5 \%)$ & 0 & 22 \\
\hline $\begin{array}{l}\text { Análisis y mejora de } \\
\text { la accesibilidad física }\end{array}$ & $11(50 \%)$ & $10(45 \%)$ & $1(5 \%)$ & 0 & 0 & 22 \\
\hline $\begin{array}{l}\text { Análisis y mejora de } \\
\text { la accesibildiad de la } \\
\text { información }\end{array}$ & $12(55 \%)$ & $10(45 \%)$ & 0 & 0 & 0 & 22 \\
\hline
\end{tabular}

Posteriormente, en las pruebas objetivas de una asignatura de Terapia Ocupacional, se incluyeron cinco ítems que evaluaban conocimientos sobre temáticas relacionadas con el diseño para todas las personas. El promedio de aciertos de esos ítems en el examen fue del $73.2 \%$ y tres de ellas fueron contestadas por el $100 \%$ de los estudiantes.

\section{Conclusiones}

En términos generales, se considera que las actividades diseñadas e implementadas han conducido a una mejora en la adquisición de competencias sobre diseño para todas las personas en los estudiantes de las titulaciones que han participado en la experiencia. Aunque se han tenido dificultades para terminar el diseño de tareas con carácter más interactivo, las actividades que sí se diseñaron por completo y se pusieron en funcionamiento lo hicieron de forma adecuada, fueron bien valoradas por los estudiantes que participaron y hay indicios indirectos de su impacto en las actitudes y conocimientos de los estudiantes en relación al diseño para todas las personas.

Respecto al impacto sobre la docencia se cree que las experiencias descritas pueden servir como guía de buenas prácticas para otras asignaturas y titulaciones, y podrían utilizarse como ejemplo de medida positiva para la mejora de la calidad de las titulaciones en lo que se refiere al ajuste a la normativa vigente sobre la atención a la diversidad en el sistema educativo 
universitario. Se considera que actividades como las descritas, serían aplicables, con pocas modificaciones, a estudiantes de otras titulaciones.

\section{Referencias}

BARROS, R., MENDES, R., PRADO, R., y MACHADO, J. (2011). Oral health and oral motor function in children with cerebral palsy. Special Care in Dentistry, 31, 58-62

BEALS, D., WONG, P.M, ALLEN, B., y RUTTER, B.(1999) Grip architecture in manual tooth brushing. Journal of Dental Research, 78, 2457.

CHRISTOPHERSEN, J. (Ed.). (2002). Universal Design: 17 Ways of Thinking and Teaching. Norway: Husbanken.

COLLINS, B. (2014). Universal design for learning: What occupational therapy can contribute. Occupational Therapy Now, 16(5), 22-23.

DEJAGER, A., y LONNAY, I. (2015). How to integrate client-centred co-design in a current curriculum: a good practice. Paper presented at the 21st Annual Meeting of ENOTHE, October, Bulgaria.

DÍEZ, E. (2013). Accesibilidad y Diseño Universal. En Miguel Ángel Verdugo \& Robert Schalock (Eds.): Discapacidad e Inclusión: Manual para la docencia (pp. 405- 421). AMARÚ, Salamanca.

DÍEZ, E., DELGADO, M. L., JIMÉNEZ, E., LEAL, E., MORENO, R., POUSADA, T., BARRAGÁN, J. A., DÍAZ, M., LÓPEZ, J. L., y SÁNCHEZ, S. (2016). Formación curricular en diseño para todas las personas en Terapia Ocupacional / Curricular Training in Design for All in Occupational Therapy. CRUE Universidades Españolas / Fundación ONCE / Real Patronato sobre Discapacidad (ISBN: 97884-617-6434-1).

DÍEZ, E., POUSADA, T., y PEREIRA, J. (2013). Productos y Tecnologías de Apoyo. En Miguel Ángel Verdugo \& Robert Schalock (Eds.): Discapacidad e Inclusión: Manual para la docencia (pp. 423441). AMARÚ, Salamanca.

DYER, M., CALLANAN, M., y FENNELL, A. (2010). Integrating Universal Design Content in Third Level Curriculum. National Disability Authority, Centre for Excellence in Universal Design, James Hubbard.

LARKIN, H., HITCH, D., WATCHORN, V., ANG, S. y STAGNITTI, K. (2013). Readiness for interprofessional learning: A crossfaculty comparison between architecture and occupational therapy students. Journal of Interprofessional Care, Early online: 1-7. doi; 10.3109/1356118202013.779233

LID, I. M. (2014). Universal Design and disability: an interdisciplinary perspective. Disability and Rehabilitation, 36, 1344-1349. doi: 10.3109/09638288.2014.931472

LIU, L. (2014). Evolution of universal design in the context of occupational therapy practice. Occupational Therapy Now, 16(5), 3-4.

MARULANDA, J., BETANCUR, J. D., ESPINOSA, S., GÓMEZ, J. L., y TAPIAS, A. (2011). Salud oral en discapacitados. Revista CES Odontología, 24 (1): 71-76. 
Propuesta de Innovación docente para el aprendizaje Interdisciplinar sobre Diseño para todas las personas entre estudiantes de los Grados de Odontología y de Terapia Ocupacional de diferentes Universidades españolas

MEDEIROS, A., BRINDEIRO, D., FEITOSA, V., y WILNEY, W. (2011). O Acesso ao Cuidado em Sáude Bucal para Crianças com Deficiência Motora: Perspectivas dos Cuidadores. Pesquisa Brasileira em Odontopediatria e Clinica Integrada Journal, 11, 593-599.

MIRÓN, J. A., COCO, M. B., ALONSO, M., MALDONADO, M. J., e IGLESIAS, H. (2014). Formación curricular en diseño para todas las personas en Medicina / Curricular Training in Design for All in Medicine. CRUE Universidades Españolas / Fundación ONCE / Real Patronato sobre Discapacidad.

MORROW, R. (2002). Building and Sustaining a Learning Environment for Inclusive Design: A Framework for teaching inclusive design within built environment courses in the UK. Online. Centre for Education in the Built Environment.

PREISER, W., y SMITH, K. H. (2011). Universal Design Handbook, 2nd edition. New York: McGrawHill.

RAO, D., AMITHA, H., y KISHAN, A. (2005). Oral higiene status of disabled children and adolescents attending special schools of South Canara, India. Hong Kong Dental Journal, 2, 107-113.

RINGAERT, L. (2002). Universal design and occupational therapy. Occupational Therapy Now, 4(5), 28-30.

SÁNCHEZ, S. y DÍEZ, E. (2013). La educación inclusiva desde el currículum: el Diseño Universal para el Aprendizaje. En H. Rodríguez Navarro y L. Torrego Egido (Eds.): Educación inclusiva, equidad y derecho a la diferencia (pp. 107-119). Las Rozas, Madrid: Wolters Kluwer España.

SÁNCHEZ, S. y DÍEZ, E. (2016). La implementación de contenidos sobre diseño universal en ingeniería informática y arquitectura en España. Psychology, Society \& Education, 8, 53-64.

SHAW, L., SHAW, M., J, y FOSTER, T., D. (1989) Correlation of manual dexterity and comprehension with oral hygiene and periodontal status in mentally handicapped adults. Community Dental Oral Epidemiology, 17(4), 187-9.

STEINFELD, E. y MAISEL, J. (2012). Universal design: Creating inclusive environments. Hoboken, NJ: Wiley \& Sons, Inc.

WATCHORN, V., LARKIN, H., ANG, S., y HITCH, D. (2013). Strategies and effectiveness of teaching universal design in a cross-faculty setting. Teaching in Higher Education, 18(5), 477-490. doi: $10.1080 / 13562517.2012 .752730$

WELCH, P. (1995). Strategies for Teaching Universal Design. Boston: Adaptive Environments. 